\title{
Gc-Ms Analysis Of Root And Aerial Parts Ethanolic Extract Of Phyllanthus Vasukii Parthipan Et Al., Sp. Nov. (Phyllanthaceae)
}

\author{
Jemimma, H. L*., Arumugasamy,K , Nantha Kumar. R. , Abdul Kaffoor H., \\ Department of Botany, Kongunadu Arts and Science College (Autonoumous), Coimbatore, Tamilnadu,
} India

\begin{abstract}
Objective: The objective of this research is to determine the possible bioactive components of the root and aerial parts of Phyllanthus vaukii using GC-MS analysis.

Methods: The GC-MS analysis of these extracts were performed using a Perkin-Elmer GC Clarus 500 system and Gas chromatograph interfaced to a Mass spectrometer (GC-MS) equipped with a BR-5MS, fused silica capillary column $(30 \mathrm{~mm} \times 0.25 \mathrm{~mm} 1 \mathrm{D} \times 0.25 \mu \mathrm{Mdf}$, composed of $5 \%$ Diphenyl $/ 95 \%$ Dimethyl poly siloxane).

Results: From $P$. vasukii root and aerial parts, thirty (30) and twenty seven (27) components were identified respectively. Of the 30 compounds eluted from the root extract, stigmasterol had the highest peak area of $18 \%$ and the lowest was n-Propyl 11-octadecenoate showing $0.38 \%$. In the aerial extract 1,2,3-Benzenetriol had the highest peak area of $31.65 \%$ and Z,Z-3,15-Octadecadien-1-ol acetate with the lowest peak area of $0.19 \%$. These results indicate the ethanol extract of $P$. vasukii aerial and root parts possess potent antioxidant, hepatoprotective, anti-inflammatory, antiarthritic, antioxidant, anticancer, Immunostimulant, antitumour, cancer preventive, antiarthritic, antidiabetic, antimicrobial effects so that it can be recommended as a plant of pharmaceutical importance.
\end{abstract}

Conclusion: However, isolation of individual phytochemical constituents may proceed to find a novel drug or lead compound.

Keywords: Phyllanthus vasukii (P.vasukii), Gc-Ms Analysis, Bioactive Compound, root and aerial parts, ethanolic extract

\section{Introduction}

The use of plants as medicines by man has been in existence since a long time and we still continue to search for plants as drug for a particular disease. Herbal medicines are safe than synthetic medicines because the phytochemicals in the plant extract target the biochemical pathway ${ }^{1}$. The plant Phyllanthus vasukii belongs to the family Phyllanthaceae. The genus Phyllanthus is large and distributed widely in tropical and subtropical countries of the world. It has been in use as herbal medicine for a long time in China, India, Brazil and South-East Asian nations. The beneficial medicinal effects of plant materials are the nature of secondary metabolites for an example, alkaloids, flavonoids, lignin, phenols and terpenes The approval of traditional medicine as an alternative form of health care and the improvement of microbial resistance to the existing antibiotics has lead researchers to scrutinize the antimicrobial compounds ${ }^{2}$. The medicinal actions of plants unique to particular plant species or groups are consistent with the concept that the combination of secondary products in a particular plant is taxonomically distinct ${ }^{3,4}$. These are used in traditional medicine practices in particularly, antibacterial, hepatoprotective, antidiabetic, antihypertensive, analgesics, antiinflammatory, hepatoprotective and antimicrobial properties ${ }^{5}$. Screening active compounds from plants has lead to the invention of new medicinal drugs which have efficient protection and treatment roles against various diseases including cancer and alzheimer's disease ${ }^{6,7}$. The Phyllanthus species are employed by the 
local people of Thailand, Latin America and Africa to cure jaundice, renal calculi and malaria etc. ${ }^{8-10}$. More than several hundreds of phytoconstituents were reported from different species of Phyllanthus, which mainly constitute lignins, triterpenoids, flavonoids and tannins. GC-MS is the best technique to identify the bioactive constituents of long chain hydrocarbons, alcohols, acids esters, alkaloids, steroids, amino acid and nitro compounds ${ }^{11}$.

\section{Material and methods}

\section{Collection of Plant material}

Roots of plant Phyllanthus vasukii was collected from P. vellore, Namakkal District, Eastern Ghats, Tamilnadu, India. The plant was described by Parthiban et al. (2017). A voucher specimen was deposited at the Herbarium of Botany Department, Bharathiar University, Coimbatore, India.

The roots and healthy aerial parts (without seed) of $P$. vasukii species were collected from Namakkal,Southern Eastern Ghats of Tamil Nadu state, Republic of India. They were thoroughly washed there itself with running water, brought to lab and shade dried. The plant was identified by Prof. Rajendran at the department of Botany, Bharathiar University, Coimbatore, India and authenticated. The voucher specimens were deposited at the Herbarium of Botany Department, Bharathiyar University, Coimbatore.

\section{Preparation of powder and extracts}

The shade dried materials were pulverized separately to fine powders. These powders were defatted with petroleum ether and then extracted with ethanol in a soxhlet apparatus. After cooling, the extracts were evaporated to dryness and kept under refrigeration for further study.

\section{GC-MS Analysis}

The GC-MS analysis of these extracts were performed using a Perkin-Elmer GC Clarus 500 system and Gas chromatograph interfaced to a Mass spectrometer (GC-MS) equipped with a BR-5MS, fused silica capillary column $(30 \mathrm{~mm} \times 0.25 \mathrm{~mm} \mathrm{1D} \times 0.25 \mu \mathrm{Mdf}$, composed of $5 \%$ Diphenyl / 95\% Dimethyl poly siloxane). For GC-MS detection, an electron ionization system with ionizing energy of $70 \mathrm{eV}$ was used. Helium gas $(99.999 \%)$ was used as the carrier gas at constant flow rate $1 \mathrm{mLmin}^{-1}$ and an injection volume of $2 \mu \mathrm{Lwas}$ employed (split ratio of 10:1); Injector temperature $280^{\circ} \mathrm{C}$; Ionsource temperature $250^{\circ} \mathrm{C}$. The oven temperature was programmed from $110^{\circ} \mathrm{C}$ (isothermal for $2 \mathrm{~min}$.), with an increase of $10^{\circ} \mathrm{Cmin}^{-1}$, to $200^{\circ} \mathrm{C}$, then $5{ }^{\circ} \mathrm{C} \min ^{-1}$ to $280^{\circ} \mathrm{C}$, ending with a $12 \mathrm{~min}$ isothermal at $280^{\circ} \mathrm{C}$. Mass spectra were taken at $70 \mathrm{eV}$; a scan interval of 0.5 seconds and fragments from 50 to $500 \mathrm{amu}$. Total GC running time was 40.50 minutes. The relative \% amount of each component was calculated by comparing its average peak area to the total areas, software adopted to handle mass spectra and chromatograms was a Turbomass.

\section{Identification of components}

Interpretation on mass spectrum of GC-MS was done using the database of National institute of Standard and Technology (NIST) having more than 62,000 patterns. The mass spectrum of the unknown component was compared with the spectrum of the known components stored in the NIST library. The name, molecular weight and structure of the components of the test materials were ascertained. The biological activities of the components were referred from Dr. Dukes' Ethanobotanical database.

\section{Result}

\section{GC-MS analysis}

GC-MS analysis of root and aerial parts of $P$. vasukii showed the presence of steroids, nitrogen and sulphur compounds. A total of 30 and 27 phytocompounds were detected in the ethanol extract of root and aerial parts respectively (Table 1 and 2). The first eluted compound was phenol, 2-methoxy-3-(2-propenyl) with a retention time of 7.92 in the root extract. Similarly levoglucosenone, a chiral compound eluted first with a retention time of 4.24 in the aerial extract. The major peak area of 18.95 and $14.81 \%$ were occupied by stigmasterol and $\beta$ - sito sterol respectively in the root extract. The aerial parts of $P$. vasukii ethanol extract showed that $1,2,3$ - benzene triol, a polyhydroxy phenolic compound occupied a major peak area of $31.65 \%$ 
Table1. GC-MS analysis of the root ethanol extract of $P$. vasukii

\begin{tabular}{|c|c|c|c|c|c|}
\hline $\begin{array}{c}\text { S } \\
\text { No. }\end{array}$ & RT & Name of the compound & $\begin{array}{l}\text { Molecular } \\
\text { Formulae }\end{array}$ & $\begin{array}{l}\text { Molecular } \\
\text { Weight }\end{array}$ & $\begin{array}{c}\text { Peak } \\
\text { Area } \%\end{array}$ \\
\hline 1. & 7.92 & Phenol, 2-methoxy-3-(2-propenyl)- & $\mathrm{C}_{10} \mathrm{H}_{12} \mathrm{O}_{2}$ & 164 & 12.01 \\
\hline 2. & 9.94 & $\begin{array}{l}\alpha \text {-D-Glucopyranoside, methyl 2-(acetylamino)-2-deoxy-3-O- } \\
\text { cyclic methyl }\end{array}$ & $\mathrm{C}_{13} \mathrm{H}_{26} \mathrm{NO}_{6}$ & 331 & 0.79 \\
\hline 3. & 11.32 & $\begin{array}{l}\text { 5,6,7,8,9,10-He xahydro-9-methyl-spiro[2H-1,3-benzo xazine- } \\
4,1 \text {-cyclohexane]-2-thione }\end{array}$ & $\mathrm{C}_{14} \mathrm{H}_{23} \mathrm{NOS}$ & 253 & 0.68 \\
\hline 4. & 11.64 & Cubedol & $\mathrm{C}_{15} \mathrm{H}_{26} \mathrm{O}$ & 222 & 2.54 \\
\hline 5. & 12.40 & Phenol, 2,6-dimethoxy-4-(2-propenyl)- & $\mathrm{C}_{11} \mathrm{H}_{14} \mathrm{O}_{3}$ & 194 & 2.63 \\
\hline 6. & 15.08 & Hexadecanoic acid, methyl ester & $\mathrm{C}_{17} \mathrm{H}_{34} \mathrm{O}_{2}$ & 270 & 1.13 \\
\hline 7. & 15.52 & Dibutyl phthalate & $\mathrm{C}_{16} \mathrm{H}_{22} \mathrm{O}_{4}$ & 278 & 1.22 \\
\hline 8. & 15.85 & n-Propyl 11-octadecenoate & $\mathrm{C}_{21} \mathrm{H}_{40} \mathrm{O}_{2}$ & 324 & 0.38 \\
\hline 9. & 16.01 & Hexadecanoic acid, ethyl ester & $\mathrm{C}_{18} \mathrm{H}_{36} \mathrm{O}_{2}$ & 284 & 6.33 \\
\hline 10. & 17.39 & 9,12-Octadecadienoic acid (Z,Z)- & $\mathrm{C}_{18} \mathrm{H}_{32} \mathrm{O}_{2}$ & 280 & 1.35 \\
\hline 11. & 17.49 & 9-Octadecenoic acid (Z)-, methyl ester & $\mathrm{C}_{19} \mathrm{H}_{36} \mathrm{O}_{2}$ & 296 & 1.45 \\
\hline 12. & 18.34 & 9,12-Octadecadienoic acid (Z,Z)-, methyl ester & $\mathrm{C}_{19} \mathrm{H}_{34} \mathrm{O}_{2}$ & 294 & 2.57 \\
\hline 13. & 18.44 & Ethyl Oleate & $\mathrm{C}_{20} \mathrm{H}_{38} \mathrm{O}_{2}$ & 310 & 3.44 \\
\hline 14. & 18.83 & Octadecanoic acid, ethyl ester & $\mathrm{C}_{20} \mathrm{H}_{40} \mathrm{O}_{2}$ & 312 & 1.13 \\
\hline 15. & 20.26 & cis-13-Eicosenoic acid & $\mathrm{C}_{20} \mathrm{H}_{38} \mathrm{O}_{2}$ & 310 & 0.43 \\
\hline 16. & 21.04 & Curan, 16,17-didehydro-, (20.xi.)- & $\mathrm{C}_{19} \mathrm{H}_{24} \mathrm{~N}_{2}$ & 280 & 0.72 \\
\hline 17. & 22.32 & Dasycarpidan-1-methanol, acetate (ester) & $\mathrm{C}_{20} \mathrm{H}_{26} \mathrm{~N}_{2} \mathrm{O}_{2}$ & 326 & 0.45 \\
\hline 18. & 23.23 & Heptanoic acid, docosylester & $\mathrm{C}_{29} \mathrm{H}_{58} \mathrm{O}_{2}$ & 438 & 1.57 \\
\hline 19. & 23.83 & Z,Z-3,15-Octadecadien-1-ol acetate & $\mathrm{C}_{20} \mathrm{H}_{36} \mathrm{O}_{2}$ & 308 & 2.63 \\
\hline 20. & 24.82 & Bufa-20,22-dienolide, 3,14-dihydroxy-, $(3 \beta, 5 \beta)$ - & $\mathrm{C}_{24} \mathrm{H}_{34} \mathrm{O}_{4}$ & 386 & 1.55 \\
\hline 21. & 25.99 & Androst-4-en-9-thiocyanomethyl-11-ol-3,17-dione & $\mathrm{C}_{21} \mathrm{H}_{27} \mathrm{NO}_{3} \mathrm{~S}$ & 373 & 1.47 \\
\hline 22. & 27.28 & 9-Octadecenamide, (Z)- & $\mathrm{C}_{18} \mathrm{H}_{35} \mathrm{NO}$ & 281 & 1.54 \\
\hline 23. & 27.75 & Squalene & $\mathrm{C}_{30} \mathrm{H}_{50}$ & 410 & 2.48 \\
\hline 24. & 28.95 & 9,12,15-Octadecatrienoic acid, 2,3-bisoxy propyl ester, (Z,Z,Z)- & $\mathrm{C}_{27} \mathrm{H}_{52} \mathrm{O}_{4}$ & 496 & 1.70 \\
\hline 25. & 30.54 & 9,10-Secocholesta-5,7,10(19)-triene-3,24,25-triol, (3ß,5Z,7E)- & $\mathrm{C}_{27} \mathrm{H}_{44} \mathrm{O}_{3}$ & 416 & 1.65 \\
\hline 26. & 30.88 & Stigmasta-5,22-dien-3-ol, acetate, $(3 \beta)$ - & $\mathrm{C}_{31} \mathrm{H}_{50} \mathrm{O}_{2}$ & 454 & 3.68 \\
\hline 27. & 31.98 & $\beta$-Sitosterol acetate & $\mathrm{C}_{31} \mathrm{H}_{52} \mathrm{O}_{2}$ & 456 & 2.82 \\
\hline
\end{tabular}


Jemimma, H. L*, International Journal of Ayurvedic \& Herbal Medicine 7(4) July.-Aug.2017 (2672-2684)

28. $\quad 34.87 \quad$ Campesterol

$\mathrm{C}_{28} \mathrm{H}_{48} \mathrm{O}$

400

5.89

29. $\quad 35.59 \quad$ Stigmasterol

$\mathrm{C}_{29} \mathrm{H}_{48} \mathrm{O}$

412

18.95

30.

$37.19 \quad \beta$-Sitosterol

$\mathrm{C}_{29} \mathrm{H}_{50} \mathrm{O}$

414

14.81

Table.2 GC-MS analysis of the aerial part ethanol extract of $P$. vasukii

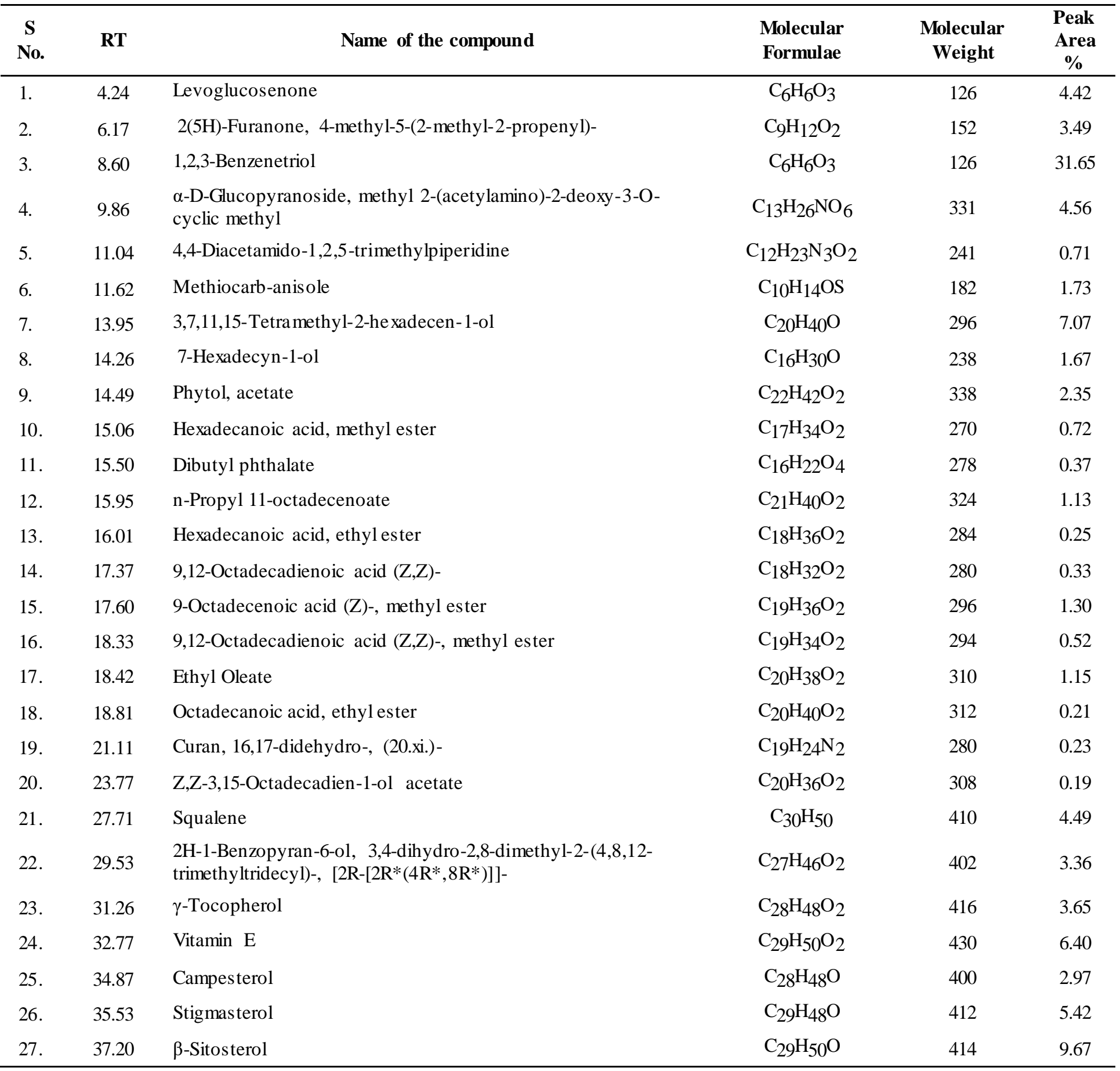

\section{Discussion}

The GC-MS chromatogram in root and aerial part ethanol extract of $P$. vasukii showed a total of 30 and 27 phytocompounds (Fig 1 and 2). The peaks in the chromatogram were compared with the database of the spectrum of known components stored in the GC-MS library. The detailed tabulation of GC-MS analysis is 
given in Table 2 and 4 . The identification of phytochemical compounds was based on the peak area, retention time, molecular weight, molecular formula and its activiy. The major bioactive compounds in the ethanol extract of P. vasukii were identified as Stigmasterol (18.95\%), $\beta$-Sitosterol (14.81\%) and Phenol, 2-methoxy-3- (2propenyl)- $(12.01 \%)$ in the root whereas the aerial parts showed the presence of 1,2,3-Benzenetriol $(31.65 \%)$ and $\beta$-Sitosterol $(9.67 \%)$.The above compounds have been reported to possess many biological activities such as antioxidant, anti-inflammatory, hepatoprotective, antiviral, antimicrobial and analgesic as referred from Dr. Duke's Phytochemical and Ethanobotanical Databases.

Table 3. Activity of bioactive compounds identified in the GCMS study of root samples of $P$. vasukii

\begin{tabular}{|c|c|c|c|c|c|c|c|}
\hline $\begin{array}{c}\text { S } \\
\text { No. }\end{array}$ & RT & $\begin{array}{l}\text { Name of the } \\
\text { compound }\end{array}$ & $\begin{array}{l}\text { Molecular } \\
\text { Formulae }\end{array}$ & $\mathbf{M} \mathbf{W}$ & $\begin{array}{c}\text { Peak } \\
\text { Area } \\
\%\end{array}$ & Compound Nature & **Activity \\
\hline 1. & 7.92 & $\begin{array}{l}\text { Phenol, 2-methoxy-3- } \\
\text { (2-propenyl)- }\end{array}$ & $\mathrm{C}_{10} \mathrm{H}_{12} \mathrm{O}_{2}$ & 164 & 12.01 & Phenolic compound & $\begin{array}{l}\text { Antioxidant Antimicrobial } \\
\text { Anti-inflammatory }\end{array}$ \\
\hline 2. & 9.94 & $\begin{array}{l}\alpha \text {-D-Glucopyranoside, } \\
\text { methyl 2-(acetylamino)- } \\
\text { 2-deoxy-3-O-cyclic } \\
\text { methyl }\end{array}$ & $\mathrm{C}_{13} \mathrm{H}_{26} \mathrm{NO}_{6}$ & 331 & 0.79 & Amino compound & Antimicrobial \\
\hline 3. & 11.32 & $\begin{array}{l}5,6,7,8,9,10- \\
\text { Hexahydro-9-methyl- } \\
\text { spiro[2H-1,3- } \\
\text { benzoxazine-4,1'- } \\
\text { cyclohexane]-2-thione }\end{array}$ & $\mathrm{C}_{14} \mathrm{H}_{23} \mathrm{NOS}$ & 253 & 0.68 & Sulfur compound & Antimicrobial \\
\hline 4. & 11.64 & Cubedol & $\mathrm{C}_{15} \mathrm{H}_{26} \mathrm{O}$ & 222 & 2.54 & $\begin{array}{l}\text { Sesquiterpene } \\
\text { alcohol }\end{array}$ & $\begin{array}{l}\text { Anti-tumor, Analgesic } \\
\text { Antibacterial, Anti- } \\
\text { inflammatory Sedative, } \\
\text { Fungicide }\end{array}$ \\
\hline 5. & 12.40 & $\begin{array}{l}\text { Phenol, 2,6-dimethoxy- } \\
\text { 4-(2-propenyl)- }\end{array}$ & $\mathrm{C}_{11} \mathrm{H}_{14} \mathrm{O}_{3}$ & 194 & 2.63 & Phenolic compound & $\begin{array}{l}\text { Antioxidant Antimicrobial } \\
\text { Anti-inflammatory }\end{array}$ \\
\hline 6. & 15.08 & $\begin{array}{l}\text { Hexadecanoic acid, } \\
\text { methyl ester }\end{array}$ & $\mathrm{C}_{17} \mathrm{H}_{34} \mathrm{O}_{2}$ & 270 & 1.13 & $\begin{array}{l}\text { Palmitic acid methyl } \\
\text { ester }\end{array}$ & $\begin{array}{l}\text { Antioxidant } \\
\text { Hypocholesterolemic } \\
\text { Nematicide } \\
\text { Pesticide } \\
\text { Lubricant } \\
\text { Antiandrogenic } \\
\text { Flavor } \\
\text { Hemolytic }\end{array}$ \\
\hline 7. & 15.52 & Dibutyl phthalate & $\mathrm{C}_{16} \mathrm{H}_{22} \mathrm{O}_{4}$ & 278 & 1.22 & $\begin{array}{l}\text { Plasticizer } \\
\text { compound }\end{array}$ & $\begin{array}{l}\text { Antimicrobial } \\
\text { Anti-fouling }\end{array}$ \\
\hline 8. & 15.85 & $\begin{array}{l}\text { n-Propyl 11- } \\
\text { octadecenoate }\end{array}$ & $\mathrm{C}_{21} \mathrm{H}_{40} \mathrm{O}_{2}$ & 324 & 0.38 & $\begin{array}{l}\text { Unsaturated } \\
\text { compound }\end{array}$ & No activity reported \\
\hline 9. & 16.01 & $\begin{array}{l}\text { Hexadecanoic acid, } \\
\text { ethyl ester }\end{array}$ & $\mathrm{C}_{18} \mathrm{H}_{36} \mathrm{O}_{2}$ & 284 & 6.33 & $\begin{array}{l}\text { Palmitic acid ethyl } \\
\text { ester }\end{array}$ & $\begin{array}{l}\text { Antioxidant } \\
\text { Hypocholesterolemic } \\
\text { Nematicide } \\
\text { Pesticide } \\
\text { Lubricant } \\
\text { Antiandrogenic } \\
\text { Flavor } \\
\text { Hemolytic }\end{array}$ \\
\hline 10. & 17.39 & $\begin{array}{l}\text { 9,12-Octadecadienoic } \\
\text { acid }(\mathrm{Z}, \mathrm{Z}) \text { - }\end{array}$ & $\mathrm{C}_{18} \mathrm{H}_{32} \mathrm{O}_{2}$ & 280 & 1.35 & Linoleic acid & $\begin{array}{l}\text { HypocholesterolemicNemat } \\
\text { icideAntiarthriticHepatopro } \\
\text { tective Anti androgenic } \\
\text { Hypocholesterolemic 5- } \\
\text { Alpha reductase inhibitor } \\
\text { Antihistaminic }\end{array}$ \\
\hline
\end{tabular}


Jemimma, H. L*, International Journal of Ayurvedic \& Herbal Medicine 7(4) July.-Aug.2017 (2672-2684)

11. $\quad 17.49$ 9-Octadecenoic acid (Z)-, methyl ester

9,12-Octadecadienoic

12. 18.34 acid (Z,Z)-, methyl ester

13. 18.44 Ethyl Oleate

14. $\quad 18.83$

Octadecanoic acid,

ethyl ester

15. 20.26 cis-13-Eicosenoic acid

16. $21.04 \quad$ Curan, 16,17-

didehydro-, (20.xi.)-

Dasycarpidan-1-

17. 22.32 methanol, acetate

(ester)

18. $\quad 23.23$

Heptanoic acid, docosyl

ester

Z,Z-3,15-Octadecadien-

1 -ol acetate

Bufa-20,22-dienolide,

20. 24.82 3,14-dihydroxy-, $(3 \beta, 5 \beta)-$

Androst-4-en-9-

21. 25.99 thiocyanomethyl-11-ol-

3,17-dione
$\mathrm{C}_{19} \mathrm{H}_{36} \mathrm{O}_{2}$

296

1.45

$\mathrm{C}_{20} \mathrm{H}_{38} \mathrm{O}_{2}$

310

3.44

$\mathrm{C}_{20} \mathrm{H}_{40} \mathrm{O}_{2}$

312

$\mathrm{C}_{20} \mathrm{H}_{38} \mathrm{O}_{2}$

310

$\mathrm{C}_{19} \mathrm{H}_{24} \mathrm{~N}_{2}$

280

0.72

$\mathrm{C}_{20} \mathrm{H}_{26} \mathrm{~N}_{2} \mathrm{O}_{2}$

326

0.45

$\mathrm{C}_{29} \mathrm{H}_{58} \mathrm{O}_{2}$

438

1.57

$\mathrm{C}_{20} \mathrm{H}_{36} \mathrm{O}_{2}$

308

2.63

$\mathrm{C}_{24} \mathrm{H}_{34} \mathrm{O}_{4}$

386

1.55

$\mathrm{C}_{21} \mathrm{H}_{27} \mathrm{NO}_{3} \mathrm{~S}$

373

1.47

AnticoronaryInsectifuge Ant ieczemicAntiacne

Oleic acid ester

Steroid

Oleic acid ester

Linoleic acid methyl ester

ond

Stearic acid ethyl
ester

Unsaturated fatty acid

Nitrogen compound

Acetate compound

Ester compound

Acetate compound

Hydroxy compound

No activity reported
Antimicrobial Antiinflammatory Anticancer Antiasthma Hepatoprotective Diuretic

Cancer preventive

Flavor

Hypocholes terolemic

5-Alpha reductase inhibitor

Antiandrogenic

Perfumery

Insectifuge

Anti-inflammatory

Anemiagenic

Dermatitigenic

Choleretic

HypocholesterolemicNemat icideAntiarthriticHepatopro tective Anti androgenic Hypocholes terolemic 5Alpha reductase inhibitor Antihistaminic AnticoronaryInsectifuge Ant ieczemicAntiacne

Cancer preventive Flavor

Hypocholes terolemic

5-Alpha reductase inhibitor Antiandrogenic Perfumery Insectifuge Anti-inflammatory Anemiagenic Dermatitigenic Choleretic

No activity reported

No activity reported

\section{Antimicrobial}

No activity reported

No activity reported

No activity reported 
Jemimma, H. L*, International Journal of Ayurvedic \& Herbal Medicine 7(4) July.-Aug.2017 (2672-2684)

22. $27.28 \quad 9-O c t a d e c e n a m i d e,(Z)-$

$\mathrm{C}_{18} \mathrm{H}_{35} \mathrm{NO}$

281

1.54

Amide compound

Linolenic acid ester

$9,12,15-$

24. 28.95 Octadecatrienoic acid,

2,3-bis oxy propylester, (Z,Z,Z)-

9,10-Secocholesta-

$25 . \quad 30.54$

5,7,10(19)-triene-

3,24,25-triol,

$(3 \beta, 5 Z, 7 \mathrm{E})-$

Stigmasta-5,22-dien-3ol, acetate, $(3 \beta)$ -

26. $\quad 30.88$

$\beta$-Sitosterol acetate

$\mathrm{C}_{31} \mathrm{H}_{52} \mathrm{O}_{2}$

456

2.82

27 31.98

$\beta$-Sitosterol acetate

Campesterol

$\mathrm{C}_{28} \mathrm{H}_{48} \mathrm{O}$

400

5.89

28

34.87 Campestero

29. $35.59 \quad$ Stigmasterol

$\mathrm{C}_{29} \mathrm{H}_{48} \mathrm{O}$

412

18.95 compound

Steroid

Antimicrobial

Anti-inflammatory

Triterpene

Antibacterial

Antioxidant

Antitumor

Cancer preventive

Immunostimulant

Chemo preventive

Lipoxygenase-inhibitor

Pesticide

Hypocholes terolemicNemat icideAntiarthriticHepatopro tective Anti androgenic Hypocholesterolemic 5-

Alpha reductase inhibitor Antihistaminic

AnticoronaryInsectifugeAnt ieczemicAntiacne

Steroid

Steroid

Steroid

Steroid

Steroid

Antimicrobial Antiinflammatory Anticancer Antiasthma Hepatoprotective Diuretic

Antimicrobial Antiinflammatory Anticancer Antiasthma Hepatoprotective Diuretic

Antimicrobial Antiinflammatory Anticancer Antiasthma Hepatoprotective Diuretic

Antimicrobial Antiinflammatory Anticancer Antiasthma Hepatoprotective Diuretic

Antioxidant Antiinflammatory Sedative Antihepatotoxic Canerpreventive Antiviral OvulantHypocholesterolemi c Estrogenic Artemicide

Antimicrobial Antiinflammatory Anticancer Antiasthma Hepatoprotective Diuretic

Table 4. Activity of bioacive compounds identified in the GCMS study of aerial part samples of $\boldsymbol{P}$. vasukii

\begin{tabular}{ccccccc}
\hline $\begin{array}{c}\text { S } \\
\text { No. }\end{array}$ & RT & Name of the compound & $\begin{array}{c}\text { Molecular } \\
\text { Formulae }\end{array}$ & M W & $\begin{array}{c}\text { Peak } \\
\text { Area } \\
\%\end{array}$ & $\begin{array}{c}\text { Compound } \\
\text { Nature }\end{array}$ \\
\hline 1. & 4.24 & Levogluctivity & Chiral & Natural product
\end{tabular}


$2(5 \mathrm{H})$-Furanone, $4-$

2. 6.17 methyl-5-(2-methyl-2propenyl)-

1,2,3-Benzenetriol

$3 . \quad 8.60$

$\alpha$-D-Glucopyranoside,

4. 9.86 methyl 2-(acetylamino)-

2-deoxy-3-O-cyclic

methyl

4,4-Diacetamido-1,2,5-

5. $\quad 11.04$ trimethylpiperidine

$6 . \quad 11.62$

Methiocarb-anisole

3,7,11,15-Tetra methyl-2-

7. 13.95 hexadecen-1-ol

7-Hexadecyn-1-ol

$8 . \quad 14.26$

Phytol, acetate

$9 . \quad 14.49$

$10 . \quad 15.06$

Hexadecanoic acid,

methyl ester

11. $\quad 15.50 \quad$ Dibutyl phthalate

n-Propyl 11-

octadecenoate

$13 . \quad 16.01$

Hexadecanoic acid, ethyl ester

$$
\mathrm{C}_{9} \mathrm{H}_{12} \mathrm{O}_{2}
$$

$$
\mathrm{C}_{6} \mathrm{H}_{6} \mathrm{O}_{3}
$$

$$
\mathrm{C}_{13} \mathrm{H}_{26} \mathrm{NO}_{6}
$$

$\mathrm{C}_{12} \mathrm{H}_{23} \mathrm{~N}_{3} \mathrm{O}_{2}$

241

0.71

$\mathrm{C}_{10} \mathrm{H}_{14} \mathrm{OS}$

$\mathrm{C}_{20} \mathrm{H}_{40} \mathrm{O}$

296

$\mathrm{C}_{16} \mathrm{H}_{30} \mathrm{O}$

$\mathrm{C}_{22} \mathrm{H}_{42} \mathrm{O}_{2}$

338

Palmitic acid methyl ester

$\mathrm{C}_{17} \mathrm{H}_{34} \mathrm{O}_{2}$

270

0.72

$\mathrm{C}_{16} \mathrm{H}_{22} \mathrm{O}_{4}$

278

0.37

$\mathrm{C}_{21} \mathrm{H}_{40} \mathrm{O}_{2}$

324

$\mathrm{C}_{18} \mathrm{H}_{36} \mathrm{O}_{2}$

284

0.25

compound

phenolic

compound

Amino
compound

Sulfur compound

\section{Terpene} alcohol

Uns aturated

alcoholic

compound

Diterpene compound

Plasticizer compound

Ester compound

Palmitic acid ethyl ester compound

synthesizing

Furan

No activity

reported

Polyhydroxy Antioxidant

Anti-

inflammatory

Analgesic

Antimicrobial

Glucose moiety

Preservative

Antimicrobial

Anti-

inflammatory

$\mathrm{C}_{18} \mathrm{H}_{32} \mathrm{O}_{2}$

280

0.33
Antimicrobial

Antimicrobial

Antiinflammatory

No activity

reported

Antimicrobial

Anti-

inflammatory

Anticancer

Diuretic

Antioxidant

Hypocholesterole mic

Nematicide

Pesticide

Lubricant

Antiandrogenic

Flavor

Hemolytic

Antimicrobial

Anti-fouling

No activity

reported

Antioxidant

Hypocholesterole mic

Nematicide

Pesticide

Lubricant

Antiandrogenic

Flavor

Hemolytic

Hypocholes terole micNematicideAn tiarthriticHepatop rotective Anti androgenic Hypocholesterole mic 5-Alpha 

$\begin{array}{ll}\text { 15. } & 17.60 \quad \begin{array}{l}\text { 9-Octadecenoic acid }(Z)-, \\ \text { methyl ester }\end{array}\end{array}$

9,12-Octadecadienoic acid (Z,Z)-, methyl ester

17.

Ethyl Oleate

$\mathrm{C}_{19} \mathrm{H}_{36} \mathrm{O}_{2}$

$\mathrm{C}_{19} \mathrm{H}_{34} \mathrm{O}_{2}$

294

0.52

$\mathrm{C}_{20} \mathrm{H}_{38} \mathrm{O}_{2}$

310

1.15

$\mathrm{C}_{20} \mathrm{H}_{40} \mathrm{O}_{2}$ ester

$\mathrm{C}_{19} \mathrm{H}_{24} \mathrm{~N}_{2}$

$\mathrm{C}_{20} \mathrm{H}_{36} \mathrm{O}_{2}$

308

Curan, 16,17-didehydro-, (20.xi.)-

$20 . \quad 23.77 \quad$ 1-ol acetate

Linoleic acid methyl ester

Oleic acid ester

Stearic acid ethyl ester

Nitrogen compound

Unsaturated alcoholic compound

Triterpene reductase inhibitor

Antihistaminic

AnticoronaryInse ctifugeAntieczemi cAntiacne

Cancer preventive Flavor

Hypocholesterole mic

5-Alpha reductase inhibitor

Antiandrogenic

Perfumery

Insectifuge

Anti-

inflammatory

Anemiagenic

Dermatitigenic

Choleretic

Hypocholesterole micNematicideAn tiarthriticHepatop rotective Anti androgenic Hypocholesterole mic 5-Alpha reductase inhibitor Antihistaminic AnticoronaryInse ctifugeAntieczemi cAntiacne

Cancer preventive Flavor

Hypocholesterole mic

5-Alpha reductase inhibitor

Antiandrogenic

Perfumery Insectifuge

Anti-

inflammatory

Anemiagenic

Dermatitigenic

Choleretic

No activity

reported

Antimicrobial

No activity

reported

Antibacterial Antioxidant

Antitumor

Cancer preventive Immunostimulant 
Jemimma, H. L*, International Journal of Ayurvedic \& Herbal Medicine 7(4) July.-Aug.2017 (2672-2684)

2H-1-Benzopyran-6-ol, 3,4-dihydro-2,8-

22. 29.53 dimethyl-2-(4,8,12-

trimethyltridecyl)-, [2R$\left.\left[2 \mathrm{R}^{*}\left(4 \mathrm{R}^{*}, 8 \mathrm{R}^{*}\right)\right]\right]-$

$\gamma$-Tocopherol

Vitamin E

24. $\quad 32.77$
$\mathrm{C}_{28} \mathrm{H}_{48} \mathrm{O}_{2}$

$\mathrm{C}_{29} \mathrm{H}_{48} \mathrm{O}$
Vitamin E compound

Chemo preventive Lipoxygenaseinhibitor

Pesticide

Flavonoid

Vitamin E compound

Antimicrobial

Anti-

inflammatory

Antiageing, Analgesic, Antidiabetic Antiinflammatory, Antioxidant, Antidermatitic, Antileukemic, Antitumor, Anticancer, Hepatoprotective, Hypocholesterole mic,

Antiulcerogenic, Vasodilator, Antis pasmodic, .Antibronchitic, Anticoronary Antiageing,

Analgesic,

Antidiabetic Antiinflammatory, Antioxidant, Antidermatitic, Antileukemic, Antitumor, Anticancer, Hepatoprotective, Hypocholesterole micAntiulceroge nic, Vasodilator, Antispasmodic, .Antibronchitic,

Anticoronary Antimicrobial Antiinflammatory Anticancer Antiasthma Hepatoprotective Diuretic

Steroid

Antioxidant Antiinflammatory Sedative Antihepatotoxic Caner-preventive Antiviral OvulantHypochol esterolemic Estrogenic Artemicide 
Jemimma, H. L*, International Journal of Ayurvedic \& Herbal Medicine 7(4) July.-Aug.2017 (2672-2684)

$\begin{array}{llllll} & & & \text { Steroid } \begin{array}{l}\text { Antimicrobial } \\ \text { Anti- } \\ \text { inflammatory }\end{array} \\ & & & & \begin{array}{l}\text { Anticancer } \\ \text { Antiasthma } \\ \text { Hepatoprotective } \\ \text { Diuretic }\end{array} \\ \end{array}$

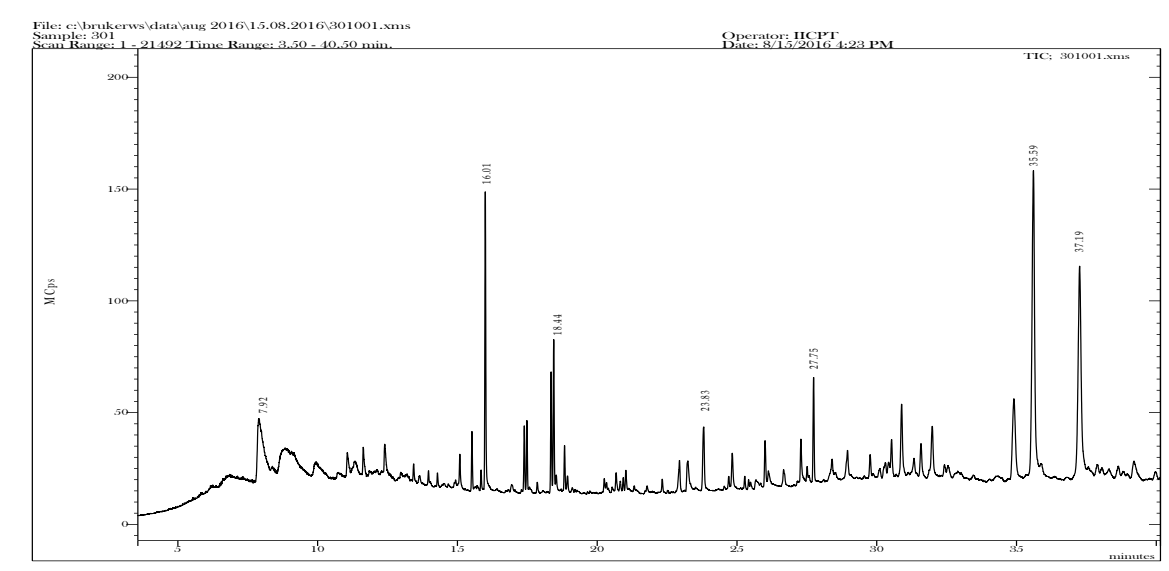

Fig. 1: GC- MS Chromatogram of $P$. vasukii root

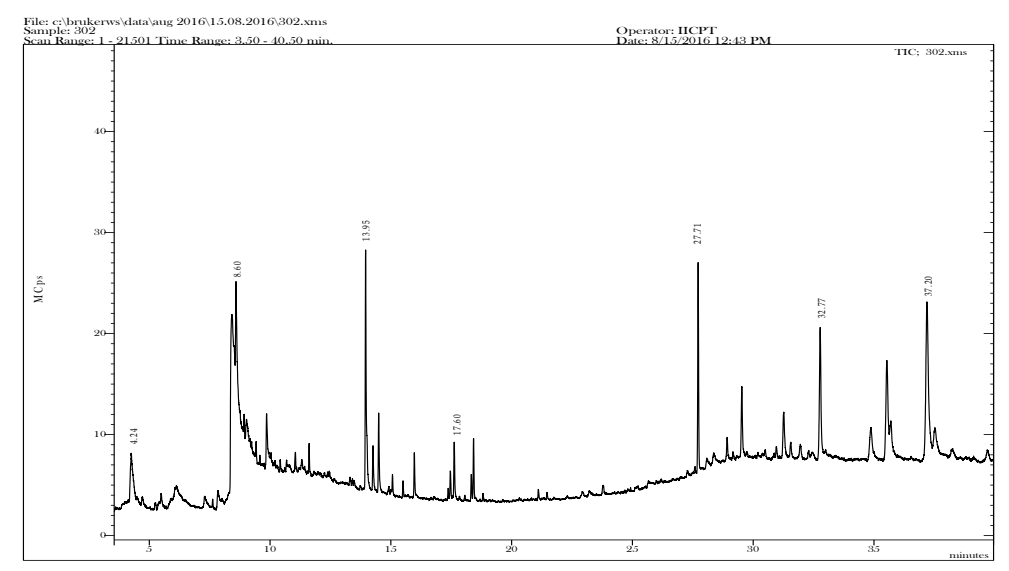

Fig. 2: GC- MS chromatogram of aerial parts of P.vasukii

The identified phytochemicals, hexadecanoic acid ethyl ester, squalene have the property of antioxidant activity12. Phenolic compounds are a class of antioxidant agents which act as free radical terminators13. Phytoconsituents such as flavonoids, terpenoids and alkaloids are known to possess hepatoprotective activity. The presence of these compounds in our extract may be responsible for its antioxidant and thus hepatoprotective activity. This activity was attributed to high reactivity of the hydroxyl groups being correlated with ROS scavenging capability14. Thus in the present study the hepatoprotective effect of ethanol extracts of P. vasukii may be due to antioxidant defense system. Thus this type of GC-MS analysis was the first step towards understanding the nature of active principles in this medicinal plant and it was helpful for further pharmacological studies. It has been reported that the bioactive components of leaves of P. amarus using GC-MS analysis and nine components from P. amarus leaves were identified 15. The prevailing components in the ethanolic extract of leaves were 3,5-di-tri-butylphenol, methyl 14-methyl 
pentadecanoate, palmitic acid (hexadecanoic acid), 10-octadecanoate, 9-hexadecenal, glycerol 1, 3dipalmitate, 2, 13-octadecadiene-1-ol, dioctytl ester and heptanoic acid (9-dece-1-yl ester). The presence of various bioactive compounds confirms the application of $\mathrm{P}$. amarus for various ailments by traditional practitioners.

Phyllanthus species have been reported to have extensive history in medicine systems 16,17 . They have listed the various classes of phytochemicals found in Phyllanthus species18. It has the maximum reports of pharmaceutically important compounds isolated from aqueous or organic solvent extracts. The lignin sphyllanthin, hypophyllanthin, niranthin, nirtetralin, virgatusin, and heliobupthalmin lactone are common to P. amarus, P. maderaspatensis, P. urinaria, and P. virgatus 19. Phyllanthin phytochemical compound which had been studied to the most extent was considered to be correlated with antiinflammatory, immunomodulatory, antitumor, and hypotensive activities20. Reports have been published on the absence of phyllanthin and hypophyllanthin from $\mathrm{P}$. maderaspatensis and $\mathrm{P}$. urinaria21. Both phyllanthin and hypophyllanthin are present in $\mathrm{P}$. amarus and $\mathrm{P}$. fraternus but the concentration of these two lignans varies substantially in the two species22. Presence of the lignan, phyltetralin, is common to P. amarus, $\mathrm{P}$. fraternus, P. maderaspatensis, P. virgatus, and P. urinaria. The lignanhinokinin has been reported from P. amarus, P. tenellus, and P. virgatus23. Flavonoids such as rutin, quercitrin, quercetin, kaempferol, and astragalin are present in both P. amarus and P. urinaria24. Presence of several ellagitannins such as geraniin, corilagin, and phyllanthusiins are also common to $\mathrm{P}$. amarus as well as $\mathrm{P}$. urinaria25.

\section{Conclusion}

In the present study, thirty and twenty nine bioactive compounds from the root and aerial parts of $P$. vasukii were identified by GC-MS analysis. The presences of various bioactive compounds justify that this plant can be used for treating various ailments. However isolation of individual phytochemical constituents and subjecting it to biological activity will definitely give fruitful results. Therefore, it could be said that $P$. vasukii contains various bioactive compounds. Hence, it is recommended as a plant of phytopharmaceutical importance. However, further studies are needed to undertake its bioactivity and toxicity profile.

\section{Reference}

1. Zaidan M, Rain N, Badrul A. In vitro screening of five local medicinal plants for antibacterial activity using disc diffusion method. Trop. Biomed. 2005; 22:165-170.

2. Parvathi S. Antimicrobial activity of some traditional medicinal plants. J. Med. Plant Res. 2010; 4:316-321.

3. Wink D, Vodovotz Y, Grisham M, DeGraff W, Cook J, Pacelli R. Antioxidant effects of nitric oxide. Methods Enzymol. 1999; 301:413-424.

4. Janakiraman N, Johnson M, Sahaya SS. GC-MS analysis of bioactive constituents of Peristrophe bicalyculata (Retz.) Nees (Acanthaceae) Asian Pac. J. Trop. Biomed 2012; S46-S49.

5. Kokwaro JO, Medicinal plants of east Africa, First Edition, East Africa Literature Bureau, Nairobo, 1976, 58-59.

6. Sheeja K, Kuttan G. Activation of cytotoxic T lymphocyte responses and attenuation of tumor growth in vivo by Andrographis paniculata extract and andrographolide. Imunopharmarcol immunotoxicol. 2007; 29:81-93.

7. Mukherjee PK, Kumar V, Houghton PJ. Screening of Indian Medicinal Plants for acetyl cholinesterase inhibitory activity. Phytother. Res. 2007; 21:1142-1445.

8. Poompachee $\mathrm{K}$ and Chudapongse N. Comparison of the antioxidant and cytotoxic activities of Phyllanthus virgatus and Phyllanthus amarus extracts. Medical Principles and Practice, 2011; 21(1): pp. 24-29.

9. Moreira J, Klein-Júnior LC, CechinelFilho V and De Campos Buzzi F. Anti-hyperalgesic activity of corilagin, a tannin isolated from Phyllanthus niruri L. (Euphorbiaceae). Journal of Ethnopharmacology, 2013;146(1): 318-323.

10. Omulokoli E, Khan B and. Chhabra SC. Antiplasmodial activity of four Kenyan medicinal plants. Journal of Ethnopharmacology, 1997;56(2):133-137. 
11. Muthulakshmi A, Margret JR, Mohan VR. GC-MS Analysis of Bioactive components of Feonia elephantum correa (Rutaceae). J. App. Pharm. Sci. 2012; 2 (2):69-74.

12. Kalay E, Yigit G, Aslan Y, Brown KE, Pohl E, Bicknell LS et al. CEP152 is a genome maintenance protein disrupted in Seckel syndrome. Nat Genet. 2011;43:23-6.

13. Om Prakash Tiwari, Yamini B, Tripathi. Antioxidant properties of different fractions of Vitex negundo Linn, Food Chem. 2007; 100(3): 1170- 1176

14. Akram, N.A., M. Ashraf and F. Al-Qurainy. Aminolevulinic acid-induced regulation in some key physiological attributes and activities of antioxidant enzymes in sunflower (Helianthus annuus L.) under saline regimes. Sci. Hort. 2012; 142: 143-148.

15. Mamza, U.T., Sodipo, O.A. and Khan, I.Z. Gas Chromatography-Mass Spectrometry (GC-MS) analysis of bioactive components of Phyllanthus amarus leaves. Int. Res. J. Plant Sci. 2012; 3 (10):pp.208-215.

16. Unander DW, Webster GL, Blumberg BS. Records of usage or assays in Phyllanthus (Euphorbiaceae). I. Subgenera Isocladus, Kirganelia, Cicca and Emblica. J Ethnopharmacol. 1990; 30: 233-264.

17. Unander DW, Webster GL, Blumberg BS. Uses and bioassays in Phyllanthus (Euphorbiaceae): a compilation II. The subgenus Phyllanthus. J Ethnopharmacol. 1991; 34: 97-133.

18. L. Nahar, S. D. Sarker, and A. Delazar, Phytochemistry of the genus Phyllanthus in Phyllanthus Species. Scientific Evaluation and Medicinal Applications, R. Kuttan and K. B. Harikumar, Eds., , Taylor and Francis Group, CRC Press, London, UK, 2011. pp. 119-138

19. K. Shanker, M. Singh, V. Srivastava, R. Verma, A. Gupta, and M. Gupta. Simultaneous analysis of six bioactive lignans in Phyllanthus species by reversed phase hyphenated high performance liquid chromatographic technique. Acta Chromatographica. 2011; vol. 23, no. 2, pp: 321-337.

20. D. F. P. Leite, C. A. L. Kassuya, T. L. Mazzuco et al. The cytotoxic effect and the multidrug resistance reversing action of lignans from Phyllanthus amarus. Planta Medica. 2006; vol. 72, no. 15, pp: 1353-1358.

21. S. K. Sharma, S. M. Arogya, D. H. Bhaskarmurthy, A. Agarwal, and C. C. Velusami. Hepatoprotective activity of the Phyllanthus species on tert-butyl hydroperoxide (t-BH)-induced cytotoxicity in HepG2 cells. Pharmacognosy Magazine. 2011; vol. 7, no. 27, pp: 229-233.

22. K. Tripathi, R. K. Verma, A. K. Gupta, M. M. Gupta, and S. P. S. Khanuja. Quantitative determination of phyllanthin and hypophyllanthin in Phyllanthus species by high-performance thin layer chromatography. Phytochemical Analysis. 2006; vol. 17, no. 6, pp: 394-397.

23. Huang ST, Yang RC, Yang LJ, Lee PN, Pang. JHS: Phyllanthus urinaria triggers the apoptosis and Bcl-2 down-regulation in Lewis lung carcinoma cells. Life Sci 2003;72: 1705-1716.

24. T. K. Nara, J. Glyeye, E. L. Cerval et al., Flavonoids of Phyllanthus niruri, Phyllanthus urinaria, Phyllanthus orbiculatus. Plantes Médicinales et Phytothérapie. 1977; vol. 11: pp: 82-86.

25. L. Yeap Foo and H. Wong. Phyllanthusiin D, an unusual hydrolysable tannin from Phyllanthus amarus. Phytochemistry. 1992; vol. 31, no. 2: pp: 711-713. 